\title{
Leopardi il filosofo poetante
}

\author{
Sandra Dugo \\ Università degli Studi di Roma "Tor Vergata" \\ sndugosan@gmail.com
}

\begin{abstract}
Proponiamo uno studio interpretativo del pensiero poetante di Giacomo Leopardi, approfondendo l'analisi filologica di alcuni versi poetici e di alcune riflessioni estratte da Pensieri di varia filosofia e di bella letteratura, conosciuti come Zibaldone. Il nostro Recanatese ha creato un'originale teoria di filosofia sull'esistenza umana, sulla vita, e sul metodo più adatto per intraprendere la ricerca della felicità, desiderio inarrestabile dell'uomo di ogni epoca. Non c'è alcun dubbio nel definire Leopardi filosofo esistenzialista. Anticipatore del nichilismo di Friedrich Nietzsche, riesce a proporre una filosofia i cui concetti principali sono: il nulla nichilistico, la ragione umana, la verità, la teoria della felicità, accogliendo l'interpretazione del filosofo italiano Emanuele Severino, del critico letterario Antonio Prete. L'obiettivo di questo studio non è esaustivo in questa sede, ma vuole dimostrare che l'interpretazione tradizionale del pessimismo individuale e cosmico del poeta è assolutamente riduttiva e insufficiente per comprendere la base teorica delle riflessioni di tutte le opere leopardiane. Lo Zibaldone non va letto come un insieme di pensieri confusi, privi di una connessione logica, perché sono in realtà un sistema di riflessioni basate su dimostrazioni certe, alla cui base vive uno scetticismo ragionato e dimostrato con una grande forza psichica in ogni sua pagina.
\end{abstract}

Parole-chiave: Giacomo Leopardi. Pensiero poetante. Filosofia di vita. Ricerca della felicita. Nichilismo.

RESUMO: O presente trabalho propõe um estudo interpretativo do pensamento poético de Giacomo Leopardi, aprofundando a análise filológica dos versos poéticos e dos conceitos filosóficos extraídos da Pensamentos de varia Filosofia e bela Literatura, que é o conhecido como Zibaldone. O poeta de Recanati criou uma teoria filosófica da existência humana, sobre a vida e sobre o método mais adapto de apresentar a teoria da busca da felicidade, desejo do homem de todas as épocas. Não há dúvida em definir Leopardi um filósofo existencialista da condição humana, antecipador do niilismo de Friedrich Nietzsche e de alguns conceitos do movimento filosófico e literário pertencente aos séculos XIX e XX. De fato, o pensamento filosófico dele propõe conceitos específicos e essenciais: o "nada niilista", a razão humana, a verdade, a teoria da felicidade, segundo a 
interpretação do filósofo italiano Emanuele Severino e do crítico literário Antonio Prete. O objetivo deste estudo não é exaustivo, mas pretende demonstrar que a interpretação tradicional do pessimismo individual e cósmico do poeta é absolutamente redutora e insuficiente para compreender o fundamento teórico das reflexões de todas as obras de Leopardi. O Zibaldone não deve ser interpretado como um conjunto de pensamentos confusos, desprovidos de uma conexão lógica, porque são um sistema de conceitos baseados na demonstração exata perfeita e precisa do pensamento dele. Tratase de uma filosofia poetante que vive num ceticismo fundamentado e demonstrado com grande força psíquica em cada página das obras.

Palavras-chave: Giacomo Leopardi. Pensamento poetante. Filosofia da vida. Busca da felicidade. Niilismo.

ABSTRACT: We propose an interpretative study of Giacomo Leopardi's poetic thought, deepening the philological analysis of some poetic verses and some reflections extracted from Thoughts of various philosophy and beautiful literature, known as Zibaldone. Our Recanatese has created an original philosophical theory on human existence, on life, and on the most suitable method for embarking on the pursuit of happiness, an unstoppable desire of man of every age. There is no doubt in defining Leopardi an existentialist philosopher. Anticipator of Friedrich Nietzsche's nihilism, he manages to propose a philosophy whose main concepts are: nihilistic nothingness, human reason, truth, the theory of happiness, welcoming the Italian philosopher Emanuele Severino's interpretation. The objective of this study, certainly not exhaustive now, is demonstrate that the traditional interpretation of the poet's individual and cosmic pessimism is absolutely reductive and insufficient to understand the theoretical basis of the reflections of all Leopardi's works. The Zibaldone should not be read as a set of confused thoughts, devoid of a logical connection, because they are in reality a system of reflections based on certain demonstrations at the base of which lives a reasoned and demonstrated skepticism with great psychic strength in every page.

Keywords: Giacomo Leopardi. Poetic thought. Philosophy of life. The search for happiness. The nihilism.

\section{Introduzione}

$\mathcal{L}$ 'idea che la filosofia possa essere separata dall'interpretazione del testo narrativo è una creazione moderna sconosciuta agli studiosi 
italiani. La nostra tradizione culturale non ha mai stabilito il confine di separazione fra l'interpretazione critico-letteraria e quella filosofica del testo narrativo o poetico, aprendo così ampi orizzonti di studio e assicurando l'approfondimento dello studio analitico, filologico e interpretativo del testo. Essere legati alla tradizione è la metodologia di studio che consente di comprendere completamente il testo di un autore vissuto in un'altra epoca e di poter approfondire il suo pensiero, perché è impossibile scrivere senza una riflessione di carattere esistenziale o filosofico. Stabilire un confine separatorio tra letteratura, poesia e filosofia è riduttivo. L'interpretazione filosofica del testo è imprescindibile per comprendere Giacomo Leopardi, soprattutto perché non separava la creatività poetica e narrativa dal proprio pensiero filosofico. Si tratta di un filosofo sui generis, tanto che emerge un pensiero filosofico poetante dall'analisi delle sue opere. L'interpretazione dell'uomo pessimista in crisi depressiva è ormai superata da tempo e attualmente non è più oggetto di studio in nessun Paese europeo e nemmeno in Italia. Pensare al poeta pessimista è un errore di interpretazione, in realtà il pensiero poetante di Leopardi indaga il mistero della dell'esistenza umana, riflettendo sulla teoria della ricerca della felicità, erede di teorie già diffuse nella cultura illuministica, e nei manuali religiosi e laici che presentavano la trattatistica della tecnica della felicità per affrontare la condizione umana e le difficoltà della vita.

L'interesse di Leopardi per la filosofia è importante per comprendere le scelte dei suoi studi, traduceva le opere di filosofia morale dei pensatori greci appartenenti al periodo ellenistico. Si pensi alla traduzione dei Caracteres (I Caratteri) di Teofrasto, all'Enchereidion di Epitteto, ai Dialoghi di Isocrate, opere che voleva pubblicare in un libro 
per l'editore Stella, ma il progetto non si realizzò e Leopardi citò alcuni brani delle sue traduzioni nella Comparazione delle sentenze di Bruto minore e di Teofrasto vicini a morte (1822), inserendo questa ultima nelle Operette morali. Era decisamente uno studioso di grande levatura che trascorreva l'intera vita nella biblioteca della propria casa, studiando senza mai fermarsi i filosofi greci che tanto lo interessavano, in particolare lo Stoicismo e l'Epicureismo.

\section{Leopardi il primo filosofo nichilista della storia}

Da anni l'interpretazione del pensiero filosofico di Leopardi ha impegnato molti studiosi italiani e europei. Tra questi cito Antonio Prete, Sebastiano Timpanaro e Emanuele Severino che ha approfondito il pensiero filosofico di Leopardi, proponendo una nuova analisi interpretativa ${ }^{1}$. Il filosofo italiano ha pubblicato numerosi saggi sul tema dell'ontologia, cioè sullo studio dell'Essere, ritenendo Leopardi l'anticipatore del nichilismo di Friedrich Nietzsche e dell'esistenzialismo ontologico e fenomenologico di Martin Heidegger.

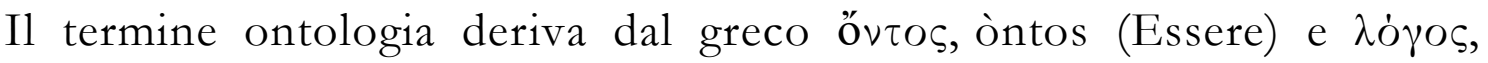
lògos (Pensare), e significa appunto pensare l'Essere ${ }^{2}$, un tema che potremmo interpretare come l'indagine esistenziale per chiarire il nostro "stare al mondo", da dove veniamo e il perché della nostra esistenza. L'analisi filosofica del pensiero poetante di Leopardi inizia con la lettura di I Pensieri di varia Filosofia e di bella Letteratura, il titolo principale scelto da lui; solo nella seconda pagina si legge "Indice del mio Zibaldone di Pensieri, cominciato agli undici di Luglio del 1827, in Firenze" Giacomo Leopardi", Il titolo Lo Zibaldone venne scelto dai posteri, sostituendolo a Pensieri. 
Severino contesta e annulla definitivamente l'interpretazione del poeta pessimista individuale e cosmico, e riflette invece sul pensiero poetante; vi è una considerevole bibliografia che nega l'interpretazione pessimistica. Il Leopardi di Severino si interroga ponendo interrogativi sulla natura e sull'esistenza umana. L'ipotesi della filosofia esistenzialista è negata da Severino, convinto assertore che questo tipo di interpretazione sia fuorviante per la comprensione del pensiero filosofico leopardiano ed esclude il confronto con Arthur Schopenhauer.

Asserire che esiste un pessimismo cosmico e individuale di fondo fa dimenticare l'aspetto filosofico. Si pensi ai numerosi studi dei critici letterari italiani che hanno proposto il superamento di queste interpretazioni tradizionali; a tale proposito citiamo Walter Binni, che è uno dei primi fra i contemporanei ad evidenziare l'aspetto filosofico dell'intellettuale che vuole lottare contro l'impotenza dell'uomo di fronte alla potenza della natura. Altri critici letterari hanno individua to una nuova via interpretativa del pensiero filosofico leopardiano, tra questi cito Cesare Luporini (2018). Quindi l'interpretazione del pessimismo cosmico è insoddisfacente e fuorviante, ma occorre dimostrarlo. Luporini approfondisce l'atteggiamento contestativo e energico del poeta rispetto alla barbarie e al dispotismo della società in cui viveva, contraddicendo appunto le teorie sul poeta pessimista deluso e depresso, di derivazione ottocentesca, risultato di una mentalità piuttosto conservatrice, tanto che lo stesso Leopardi espresse il suo disappunto più volte nelle lettere dell'Epistolario.

Nel saggio Leopardi Progressivo Cesare Luporini ipotizza che le opere di Leopardi sono anticipate in buona parte nell'officina dei Pensieri (Zibaldone), costringendo il lettore in un naufragio immaginario. Binni 
ci indica un'interpretazione contraddittoria al tradizionale pessimismo, definendo Leopardi "maestro di vita ottimistico [...], filosofo combattente" (1984) per quanto riguarda l'aspetto sociale e politico del pensiero, e ancora presentandoci il pensiero leopardiano come la migliore espressione del dell'esistenzialismo italiano. A questo proposito leggiamo i bei versi del Canto La ginestra il fiore del deserto che cresce spontaneo nelle zone vulcaniche dopo le eruzioni e appare con grande vigore di fronte alla potenza distruttrice del vulcano Vesuvio fino all'ultimo istante di vita nei versi 304-310.

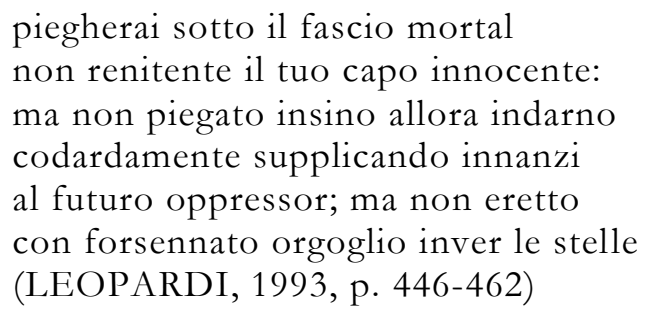

Questi versi mostrano il coraggio e l'ardimento di Leopardi e ritengo che la sua poesia rivela il desiderio di sfidare la potenza della natura fino all'ultimo estremo tentativo, cercando di contrastare la sua forza distruttrice.

Severino propone un'interpretazione diversa e la sua lettura è una esegesi filosofica, presentando la visione filosofica del pensiero ontologico del poeta di Recanati. Il concetto del "nulla" è il tema centrale delle riflessioni, soprattutto dei Pensieri, conosciuti come Zibaldone. Leopardi è il precursore del nichilismo attraverso le opere in versi e le riflessioni filosofiche dei Pensieri. I concetti filosofici espressi tra le righe dal poeta sono le basi per lo sviluppo del nichilismo dei filosofi novecenteschi, come, per citarne alcuni, Nietzsche. Anche se non esiste un concetto comune del nichilismo in Leopardi con il 
filosofo tedesco, è noto che Nietzsche conosceva Leopardi e lo aveva commentato spesso.

Il "concetto del nulla" è analizzato da Severino nel saggio Il nulla e la poesia in cui il poeta Recanatese è definito il pensatore che anticipa il nichilismo di Nietzsche e di Heidegger; il pensiero poetante è espresso attraverso i versi dei Canti, nei dialoghi delle Operette morali e nei Pensieri dello Zibaldone mediante suggestive immagini poetiche. La ragione scopre la verità dell'esistenza, ed è verissima pazzia: la precarietà esistenziale è l'incapacità dell'uomo di opporsi alla potenza della natura, l'espressione indica il ciclo della vita che scorre in un flusso continuo di nascita e di morte, di rigenerazione e di annientamento. Per Leopardi il nulla è l'origine del divenire, cioè del ciclo vitale di ogni essere vivente, e anche dell'Ente creatore dell'universo, che non va interpretato necessariamente con il Dio Cristiano, perché è definito più volte come un principio creatore dell'universo, un Ente sconosciuto e ignoto alla comprensione umana. Il 18 luglio 1821 scrive nel Pensiero 1341 del primo volume dei Pensieri nell'edizione di Giuseppe Pacella: "In somma il principio delle cose, e di Dio stesso, è il nulla. Giacché nessuna cosa è assolutamente necessaria, cioè non v'è ragione assoluta perch'ella non possa non essere, o non essere in quel tal modo ec." (LEOPARDI, 1991, p. 814). Il significato è presto chiarito: la vita dell'universo è inserita in un ciclo continuo e infinito dentro il quale ogni essere vivente e non vivente (ogni cosa) nascono, muoiono, rinascono e cambiano senza necessariamente permanere nello stesso stato.

L'uomo si trova immerso in questo ciclo che cambia continuamente e che è l'ordine naturale della vita e della morte del movimento continuo vitale di creazione e annientamento la cui origine è il nulla. Severino ritiene che Leopardi ha individuato un concetto 
filosofico fondamentale già presente nella filosofia greca: "Leopardi riesce a pensare e a dire che, poiché le cose si annullano ed escono dal nulla, esse sono nulla. Riesce a raggiungere il pensiero essenziale dell'Occidente, il pensiero che sorregge l'intera storia della nostra civiltà e della nostra cultura". (SEVERINO, 2005, p. 27. Corsivo dell'autore).

Leopardi sa ascoltare l'Essere, come non seppero farlo Omero, né Anassimandro e né Eraclito; l'errore dell'uomo è negare che il movimento universale sia eterno e immutabile e che abbia un inizio e una fine, contraddistinto dalla nascita e dalla morte, dentro il quale la vita compare e scompare. Leopardi scopre l'errore d'Occidente, perché più volte cita il pensiero degli antichi Greci i quali esprimevano il concetto dell'Essere integralmente con il linguaggio poetico, a differenza dei poeti medievali come Dante Alighieri. Eraclito di Efeso scriveva che l'universo scorre incessantemente (panta rei: tutto scorre), rappresentando così il concetto del "divenire" (l'universo in movimento che cambia), secondo il quale l'universo scorre e cambia in un ciclo continuo di nascita e morte; l'idea della vita in movimento era rappresentata dall'immagine metaforica del fiume che cambia, facendo scorrere le sue acque; è impossibile riconoscere le stesse acque che cambiano continuamente. Eraclito interrompe l'unicità dell'Essere di Parmenide, concetto che indica invece la continuità dell'universo infinito, immutabile e senza interruzioni. Il pensiero poetante di Leopardi si fa interprete dell'errore di Eraclito e dell'intera filosofia occidentale dell'epoca moderna; tuttavia cercare l'origine dell'esistenza umana e degli esseri viventi e non viventi nella fonte cosmica è un errore, perché produce dolore e infelicità nell'uomo di fronte alla morte. È il nulla, un concetto che esula dalle nostre capacità intellettive, 
non possiamo dire cosa è e se tentiamo di farlo ci perderemo nell'angoscia di non sapere dargli un significato. Non si tratta di pessimismo, ma bensì è la riflessione razionale che cerca la verità, per spiegare l'esistenza dell'universo. I concetti sono espressi con il linguaggio poetico e la riflessione filosofica diventa pensiero poetante nei Canti, quindi l'opera poetica e narrativa di Leopardi va interpretata in questa prospettiva, se si vuole comprendere l'essenza del suo pensiero. Nei lunghi anni di studio trascorsi nella meravigliosa e immensa biblioteca del padre Monaldo, Leopardi ha letto i filosofi greci, individuando in Eraclito la responsabilità di avere creato l'infelicità dell'uomo ${ }^{4}$. La filosofia eraclitea (physis) evidenzia il ruolo della natura matrigna che governa la nascita, la vita e la morte di tutti gli esseri viventi, animati e inanimati; quindi si rinvia alla physis di Eraclito. Si pensi che l'enorme erudizione di Leopardi ci costringe a indagare le fonti dei suoi studi appassionati sulla filosofia greca, definiti da lui ironicamente "uno studio matto e disperatissimo", che non ha alcun riferimento con una depressione o una debolezza psicologica. Eppure, tranne Eraclito, Leopardi ritorna all'antico pensiero dei Greci molto prima di Nietzsche. Il poeta riflette sull'esistenza, sulla vita e sull'infelicità dell'uomo, cioè sull'Essere, attraverso l'espressione poetica dei Canti, in alcuni dialoghi delle Operette morali ma soprattutto nei Pensieri, titolo principale dell'opera di Leopardi e conosciuto come Zibaldone. Ne La ginestra il vulcano, definito il "formidabil monte e sterminator Vesevo", distrugge il paesaggio circostante, bruciando anche l'ultimo fiore rimasto: la ginestra. La lava lascia dietro di sé uno scenario desolante: "campi cosparsi di ceneri infeconde, e ricoperti dell'impietrosa lava" (LEOPARDI, 1993, p. 447) ${ }^{5}$. È il deserto del nulla. 
Ma se, per Leopardi, la parte raziocinante dell'uomo scopre la terribile verità dell'esistenza umana, cioè la morte come cessazione di vita e generatrice di infelicità, la poesia è consolatrice e crea diverse immagini poetiche, quasi terapeutiche di fronte all'infelicità. Tuttavia, durante la notte, di fronte alla lava distruttrice del Vulcano Vesuvio, al di lá delle "rive desolate", lo sguardo del poeta osserva le stelle brillanti nel cielo sereno: “in purissimo azzurro veggo dall'alto fiammeggiar le stelle" (LEOPARDI, 1993, p. 455). I versi evocano un'immagine meravigliosa e consolatrice ed è frequente incontrare altre immagini simili in altri Canti, Il gallo silvestre, Alla luna, e nei Pensieri dello Zibaldone.

I primi versi del canto mostrano la condizione autentica dell'uomo di fronte al divenire, sterminatore e quindi "formidabile", radice del terrore [...] "L'arida schiena del formidabil monte" è il deserto del nulla, abitato soltanto dalla ginestra. Il monte sterminatore sta al centro di tutto il paesaggio, incombe su tutto, ma solo la ginestra lo vede da vicino, ne vede il vero volto (SEVERINO, 2005, p. 233).

Leopardi è il primo poeta occidentale che individua nella verità l'origine dell'infelicità dell'uomo, che vuole scoprire il mistero dell'esistenza umana; l'errore della filosofia dell'Occidente è pensare all'Essere diveniente, al processo del divenire della nascita della vita, alla sua fine e del suo rinascere in un continuo movimento dell'universo intero. Nel Pensiero 1341, Leopardi scrive che l'origine cosmica dell'universo e dell'Ente sconosciuto all'intelletto umano è il nulla, cioè quello che non possiamo comprendere: "il principio delle cose, e di Dio stesso, è il nulla" e aggiunge anche che "il primo ed universale principio delle cose, o non esiste, né mai fu, o se esiste o esisté, non lo possiamo in niun modo conoscere". (LEOPARDI, 1991, p. 814). Ma l'Ente non è intellegibile, non può essere compreso dall'intelletto, perché non è 
riconducibile all'esperienza umana, e deriva anch'esso dal nulla. Perciò nei Pensieri il nulla è il principio universale di tutte le cose e perfino di Dio, ricordando che è riferito all'Ente principio universale creatore e non al Dio Cristiano. Scoprire la verità nascosta dell'universo svela il processo di produzione e annientamento all'interno del quale tutte le cose nascono dal nulla e nel nulla confluiscono, coinvolte nel ciclo del divenire. Scoprire che le leggi della natura sono ostili genera nell'uomo l'infelicità. Ebbene interpretare i versi poetici come una diretta esperienza individuale di Leopardi è molto riduttivo e fuorviante rispetto al significato che è alla base del pensiero poetante e che ci conduce in un discorso molto più ampio, che in questa sede non è neppure possibile completare, tanta è la sua complessità.

Leopardi nega la filosofia dell'Iperuranio di Platone e scrive che esiste una grande forza creatrice di tutte le cose esistenti e quindi non c'è un mondo ultra-terreno a cui il nostro mondo conosciuto somigli dopo la creazione. È la teoria dell'Iperuranio immaginato da Platone. Il riferimento è il Pensiero 1340 e il 1341

\footnotetext{
supporre il bello e il buono assoluto, è tornare alle Idee ${ }^{6}$ di Platone, e risuscitare le idee innate dopo averle distrutte, giacché tolte queste, non v'è altra possibile ragione per cui le cose debbano assolutamente e astrattamente e necessariamente essere così o così, buone queste e cattive quelle, indipendentemente da ogni volontà, da ogni accidente, da ogni cosa di fatto, che in realtà è la sola ragione del tutto, e quindi sempre e solamente relativa, e quindi tutto non è buono, bello, vero, cattivo, brutto, falso, se non relativamente (LEOPARDI, 1991, p. 814).
}

Quando la verità svela la realtà materialistica che causa l'infelicità che non deriva da un pessimismo per l'esistenza umana, interpretazione riduttiva contestata più volte perfino dallo stesso Leopardi, è invece la percezione della precarietà umana soprattutto quando viene negata ogni possibilità di approdo alla certezza di fronte all'angoscia esistenziale. Il 
senso di impotenza e la relativizzazione delle cose che possono essere in un modo $\mathrm{o}$ in un altro conduce verso l'infelicità. La natura ha un potere immenso sull'uomo si pensi all'immagine poetica de La ginestra. Tuttavia "il fiore del deserto" distrutto dall'eruzione vulcanica rappresenta la speranza per il futuro e per la rinascita. La poesia è consolatrice e con le sue immagini idilliache assume una funzione terapeutica per l'angoscia esistenziale. Il pensiero poetante del Recanatese, anticipa il nichilismo almeno cento anni prima che Nietzsche formulasse le sue teorie.

Per Leopardi, l'uomo è indifeso ("ignuda natura") di fronte alla potenza della natura è incapace di cambiare il corso naturale della vita, non può comprendere il mistero dell'esistenza umana che confluisce nella fine della vita stessa "in te, morte, si posa nostra ignuda natura, lieta no ma sicura dell'antico dolor." (LEOPARDI, 1993, p. 150). L'ignuda natura è l'incapacità umana di opporsi alla morte, di svelare il mistero della nascita e della morte, ma la consapevolezza dei propri limiti non significa essere pessimisti, è invece caratteristica di razionalità e del materialismo. Le figure retoriche create da Leopardi spiegano in versi e in prosa quanto sia potente la forza che governa il processo del "divenire". Nella Palinodia al Marchese Gino Capponi narra che un fanciullo si diverte a scegliere il destino di tutte le cose e a distruggerle con un gioco crudele, "la natura crudel, fanciullo invitto / il suo capriccio adempie, e senza posa / distruggendo e formando si trastulla" (LEOPARDI, 1993, p. 434). Nel Dialogo della Natura e di un Islandese descrive la natura con le sembianze di una grande donna dall'aspetto maestoso che non si accorge affatto se sta procurando felicità o dolore agli esseri viventi. La donna gigantesca spiega all'Irlandese 
quando vi offendo in qualunque modo e con qual si sia mezzo, io non me n'avvengo, se non rarissime volte: come, ordinariamente, se io vi diletto o vi benefico, io non lo so; e non ho fatto, come credete voi, quelle tali cose, o non fo quelle tali azioni, per dilettarvi o giovarvi (LEOPARDI, 1993, p. 122).

\section{La teoria del piacere e la ricerca della felicità}

Comprendere completamente il significato del pensiero leopardiano è possibile solo se riflettiamo sul nesso tra filosofia e poesia. Dall'analisi delle opere di Leopardi emerge una poetica delle immagini sublimi e della contemplazione, di un nuovo ritrovato classicismo attraverso gli antichi Greci, nel tentativo di negare il finto paradiso promesso dalla Ragione Illuministica. E non ha senso parlare di poesia idillica del ricordo e della percezione delle illusioni senza un riferimento alla prospettiva filosofica di base. Illusioni, ricordi, immagini e percezioni della poesia consolatrice di fronte alla consapevolezza della precarietà umana, sono le caratteristiche fondamentali della poetica leopardiana di origine pre-romantica, sostenuta dalla prospettiva di tipo esistenziale. Credo che la teoria del piacere sia alla base dell'intera produzione poetica, ciò spiegherebbe la presenza contemporanea del Leopardi idillico sentimentale e romantico e del Leopardi progressivo e titanico, eroico combattivo contro lo stato di infelicità dell'uomo. A tale proposito leggiamo alcuni passi del Pensiero 646-7:

La somma della teoria del piacere, e si può dir anche, della natura dell'animo nostro e di qualunque vivente, è questa. Il vivente si ama senza limite nessuno, e non cessa mai di amarsi. Dunque non cessa mai di desiderarsi il bene, e si desidera il bene senza limiti. Questo bene in sostanza non è altro che il piacere. Qualunque piacere ancorché grande, ancorché reale, ha limiti. Dunque nessun piacere possibile è proporzionato ed uguale alla misura dell'amore che il vivente porta a se stesso. Quindi nessun 
piacere può soddisfare il vivente [...]. Perché questi desidera sempre di più, giacché per essenza si ama, e quindi senza limiti. Ottenuto anche di più, quel di più similmente non gli basta. [...] (LEOPARDI, 1991, p. 427-428).

L'infelicità deriva inizialmente dall'insoddisfazione senza limiti, per cui "il vivente $[\ldots]$ non sentendosi soddisfatto, [...] non può provar pieno piacere" (LEOPARDI, 1991, p. 427). In sostanza il Pensiero 647 e il 648 rinviano alla precarietà dell'uomo il quale, non riuscendo ad appagare se stesso, è incapace di raggiungere la felicità assoluta. Nasce allora l'esigenza di una poesia consolatoria volutamente sperimentata dal poeta per se stesso. Leopardi riflette e ragiona senza sosta sui "mezzi" utilizzati, commentando la teoria del piacere: "mezzi meno universali o durevoli o valevoli, ma pur sono gli altri da me notati, p. e. (ed è uno dei principali) lo stupore di carattere o d'indole: gli uomini così fatti sono i più felici: gli uomini incapaci di questa qualità, sono i più infelici” (LEOPARDI, 1991, p. 427). Pertanto la ricerca della felicità può contrastare il dolore derivato derivato dalla consapevolezza della propria precarietà e lo fa attraverso il bellissimo Pensiero 650, prendendo spunto da D'Alembert (Éloges de l'Académie Françoise) dove afferma che "il senso vivo del desiderio di felicità tormenta" gli uomini insoddisfatti, tuttavia può essere utile con: "impressioni straordinarie, meraviglie di qualunque sorta, $[\ldots]$ sensazioni straordinarie di qualsivoglia genere, $[\ldots]$ immaginazione, estasi che deriva dalla fantasia, e sentimento indefinito, dalla bella natura e vedi la teoria del piacere" (LEOPARDI, 1991, p. 429).

L'invenzione poetica esige la necessità di riflessioni filosofiche sui temi esistenziali ed è contemporanea all'atteggiamento titanico di Leopardi attraverso cui vuole scoprire la verità, ma lo fa terribilmente perché vede l'infelicità e la realtà di tutte le cose: il deserto della 
Ginestra è la realtà chiarificata nella sua reale totalità, completamente e senza dubbi. Dal lato opposto la poesia leopardiana consola e, riflettendo su quanto detto, scopriamo che il poeta offre un rimedio all'infelicità umana, creando immagini che sollevano l'anima dall'angoscia del nulla, per riuscire a sopravvivere nel buio nichilistico, un infinito sconosciuto per l'uomo. Si pensi allo spettacolo del niente in cui "s'annega il pensiero" de L'infinito (LEOPARDI, 1993, p. 300). Nelle Ricordanze evocare i ricordi è un modo per curare il dolore provocato dalla ragione, cioè proprio da quella razionalità che scopre la verità terribile della precarietà umana. Nel canto $A$ se stesso Leopardi medita sulle ultime illusioni pensando: "perì l'inganno estremo ch'eterno io mi credei" (LEOPARDI, 1993, p. 403). La condizione esistenziale lo spinge a vedere nella natura un "brutto potere che, ascoso a comun danno impera" è la potenza della natura misteriosa che governa tutti gli esseri viventi per causare il loro danno. Il ricordo invece è un momento piacevole che salva dall'infelicità. Leopardi comprende la condizione umana di incertezza, perché gli esseri umani sono governati dalle leggi universali della natura. L'incapacità di comprendere il significato del mistero dell'intero universo si aggiunge alla consapevolezza della precarietà del vivere nel mondo. La poesia consolatrice sceglie di evocare immagini, visioni idilliache nei Canti, in particolare ne L'infinito; il poeta si lascia trasportare nel sogno ad occhi aperti davanti a "l'ermo colle" (LEOPARDI, 1993, p. 300-301), dinanzi agli "interminati spazi" e ai "sovrumani silenzi". Quindi la "profondissima quiete" (LEOPARDI, 1993, p. 300) e "l'infinito silenzio" rappresentano un momento di contemplazione, dimenticando l'infelicità per "naufragare" dolcemente nel mare idealizzato dell'infinito (LEOPARDI, 1993, p. 301). Ma cosa è "l'infinito"? È il 
mistero, l'inconoscibile, quello che non siamo capaci di spiegare, è l'intero ciclo del divenire dello scorrere dell'universo.

Le espressioni di senso comune usate in questo contesto dal poeta evocano le visioni dell'immaginazione e dell'illusione. $\mathrm{E}$ un dolce inganno attraverso cui l'anima si illude di essere eterna: "lo spettacolo delle nullità", è l'estasi della malinconia e della disperazione? No. L'infinito è invece l'ascolto del silenzio oltre la siepe, permette di immaginare un'altra dimensione, quella dell'immaginazione.

La poesia offre la possibilità di uscire dall'angoscia esistenziale attraverso le illusioni senza le quali apparirebbe il baratro del nulla nichilistico contro cui si vuole lottare energicamente per non essere vanificati. Cosa può fare l'uomo senza l'ultima illusione che possa dargli l'unica possibilità di sognare? L'innocuo inganno dei sensi è necessario per non precipitare nell'angoscia del nulla. A tale proposito il sentimento, l'illusione e l'immaginazione sono i tre elementi fondamentali del pensiero poetante. Il sentimento del nulla nasce da una dimensione in cui l'immaginazione è la sola predominante, all'interno di una prospettiva spirituale che esclude la razionalità, perché la ragione annullerebbe l'efficacia della poesia consolatrice. Quindi il sentimento è l'illusione sono un modo estremo per evitare di precipitare nella visione della nullità delle cose.

La ragione, parte raziocinante dell'uomo, svela la "nullità degli essenti" (SEVERINO, 2005, p. 74-75), che rappresentano il nulla da cui tutte le cose nascono e confluiscono nuovamente nel ciclo del divenire. È una visione incomprensibile per l'intelletto umano, su cui si riflette nei Pensieri 103 e 104. Il mistero di questo svelamento genera l'angoscia esistenziale, perciò per Leopardi "la ragione è verissima pazzia" (LEOPARDI, 1991, p. 116-117), perché implica la follia di vedere la 
nullità delle cose, una contemplazione che vanifica se stessi; questa pazzia è "verissima" perché fa guardare la realtà delle cose, così come esse sono in una percezione vera e terribile, ma misteriosa. Nel Frammento apocrifo di Stratone da Lampsaco, l'uomo tenta di salvare se stesso dalla nullità, per "amor proprio" (LEOPARDI, 1993, p. 190194). Questo materialismo estremo del pensiero nasce dall'idea che esiste un nascere e un morire, in cui l'uomo e gli esseri viventi appartengono al ciclo dell'infinito universo. Leopardi interpreta il pensiero occidentale dopo Parmenide come la follia dell'Occidente, cioè una "verissima pazzia" (LEOPARDI, 1991, p. 116-117), anticipando il nichilismo di Nietzsche e per Severino è il baratro che l'Occidente nasconde nel proprio inconscio (SEVERINO, 2005, p. 286), in cui l'essere si annulla, all'interno del ciclo del divenire.

Per Leopardi l'intera filosofia occidentale scopre il mistero dell'esistenza e provoca l'angoscia, perché la comprensione di quello che è oltre la dimensione terrena è impossibile e non è consentito capire il mistero della vita e dell'universo. Scoprire la verità è una follia e conduce all'annientamento, cioè al nichilismo.

Raggiungere la condizione di felicità è impossibile finché domina l'uso della ragione, mentre l'illusione della poesia ispira l'immaginazione, creando un senso di appagamento e di piacere; si tratta di un innocuo inganno necessario. La metafora poetica più volte usata da Leopardi è una finzione e un lieto inganno per il lettore; pensare a una crisi esistenziale del poeta è fuorviante e impedisce la corretta interpretazione del Pensiero 106 e 107 (LEOPARDI, 1991, p. 116-117). È importante invece riflettere sugli aspetti di spiritualità e di materialismo, sull'immortalità dell'anima, sull'uso della ragione; la parte sentimentale incontra la ragione materialista, che getta l'uomo nello 
sconforto. In realtà, al di là di quanto asserisce Severino, è bene precisare che Leopardi sta vivendo in un periodo di passaggio tra l'illuminismo e il romanticismo, e la motivazione del conflitto tra sentimento e ragione va spiegato con il momento culturale, letterario, politico e sociale che lo influenza con le sue problematiche. Infatti frequenta gli ambienti culturali più disparati, ascolta i discorsi filosofici, e ha modo di riflettere sul alcuni temi discussi tra gli intellettuali e i filosofi dell'epoca nei salotti della alta borghesia e della nobiltà, e nei Caffè culturali. Senza dubbio l'aspetto romantico sentimentale convive con il materialismo settecentesco e illuminista, definitivamente, superato nell'ultima fase della produzione letteraria e poetica. Si noti la complessità di questa analisi che esula dalla semplicità di un'interpretazione che risolve velocemente l'interpretazione delle opere come risultato di un pessimismo del poeta.

\section{La noia deriva dal disagio esistenziale.}

La noia, il nulla e la morte sono tre concetti che indicano l'assenza di piacere e di felicità, e il vuoto lasciato produce la noia, più volte oggetto di riflessione nei Pensieri. Si noti che "noia" non ha il significato tradizionale che conosciamo, qui indica la condizione di vuoto dell'anima, causato dalla mancanza dell'illusione della poesia che offre invece il dolce inganno.

Chi dice assenza di piacere e dispiacere, dice noia, non che assolutamente queste due cose sieno tutt'una, ma rispetto alla natura del vivente, in cui l'una senza l'altra (mentre ch'ei sente di vivere) non può assolutamente stare. La noia corre sempre e immediatamente a riempiere tutti i vuoti che lasciano negli animi de' viventi il piacere e il dispiacere; il vuoto, cioè lo stato d'indifferenza e senza passione, non si dà in esso animo, come non si dava in natura secondo gli antichi. (LEOPARDI, 1991, p. 1939-1940). 
Quegli "antichi” sono i filosofi e i poeti greci i quali evitavano l'infelicità attraverso la poesia e la filosofia. L'assenza di passione è la mancanza di sentimenti dell'anima, confermando il Pensiero 3715 dove Leopardi scrive che la "noia" è una passione negativa: "O vogliamo dire che il vuoto stesso dell'animo umano, e l'indifferenza, e la mancanza d'ogni passione, è noia. La quale è pur passione". L'uomo desidera la felicità, perché questo sentimento consente di vivere pienamente la propria esistenza; eppure, nonostante tutto, la noia con il suo senso di vuoto, permette la rinascita del desiderio di raggiungere la felicità. "La noia è il desiderio della felicità, lasciato, per così dire, puro. Questo desiderio è passione. Quindi l'animo del vivente non può mai essere senza passione” (LEOPARDI, 1991, p. 2046). La sensazione del vuoto esistenziale spinge l'uomo a recuperare il suo cammino verso la ricerca della felicità, riflessione espressa nel Pensiero 3879

\begin{abstract}
Sono molte volte che la noia è un non so che di più vivo, che ha più sembianza perciò di passione, e quindi avviene che non sia sempre in tali casi chiamata noia, benché filosoficamente parlando, ella lo sia, consistendo in quel medesimo in cui consiste quel che si chiama noia, cioè nel desiderio di felicità lasciato puro, senza infelicità né felicità positiva e differendo solo nel grado da quella che noia comunemente è chiamata" (LEOPARDI, 1991, p. 2046)
\end{abstract}

Infatti nel Pensiero 3880 continua spiegando con più precisione i caratteri principali della sensazione negativa definita "noia"

Questa sorta di passione, diversa dalla noia comunemente detta, ma dello stesso genere ec., questa ancora io voglio comprendere sotto il nome di noia, e ad essa ancora si deve intendere ch'io abbia riguardo quando affermo che la noia corre immancabilmente e immediatamente a riempiere qualunque vuoto lasciato dal piacere o dispiacer così detto e che l'assenza dell'uno e dell'altro è noia per sua natura, e che mancando essi, v'è la noia necessariamente. (LEOPARDI, 1991, p. 2046) 
La noia rappresenta la forza che nasce della visione poetica del nulla, senza la quale non potrebbe nascere il desiderio di rinascita. Quindi il dolore e l'infelicità, sono estremi segni di vita, che si oppongono al nulla nichilistico, la loro mancanza produce la noia. Per Severino la pienezza e il vuoto rappresentano il positivo e il negativo del nichilismo. Tuttavia credo si tratti di considerazioni che Leopardi fa sull'esistenza umana, sul mistero della vita e della morte; e non c'è l'intenzione di costruire una filosofia sul nichilismo, ma vi è un tentativo di riflettere sul male di vivere. Benché l'approdo al nichilismo ci sia, è del tutto inconsapevole. La sensazione di angoscia, ricorrente nella produzione poetica, nasce dalla meditazione sul mistero dell'esistenza; Leopardi vorrebbe comprendere l'ignoto, ma come tutti gli esseri umani è incapace di opporsi al destino; in questo la forza titanica della poesia assume una funzione fondamentale. Si interroga sul mistero dell'esistenza, vuole svelare l'ignoto, ma scopre solo lo spettacolo delle nullità; nel Dialogo di Federico Ruysch e delle sue mummie, l'interrogativo finale di Ruysch ai defunti è preciso e diretto, ma non ottiene alcuna risposta: "come vi accorgeste in ultimo, che lo spirito era uscito dal corpo? Dite: come conosceste d'essere morti?” (LEOPARDI, 1993, p. 150-155). La domanda rivela l'interrogativo più inquietante dell'uomo di ogni epoca: il mistero della vita dopo la morte. Ancora una volta vengono evidenziati i limiti umani dell'incapacità di poter comprendere l'ignoto. E allora si incontra il buio totale del nulla, quello che Severino indica come il nichilismo leopardiano.

\section{La ragione e il desiderio del genio Leopardi.}

Il genio della Ginestra è il rimedio proposto all'infelicità e deve essere considerata l'ultima illusione capace di alleviare il dolore, la 
speranza per la rinascita di una nuova fase. Si è detto che la ragione non può essere un rimedio per l'infelicità e che non può risolvere l'angoscia del nulla, ma rappresenta il contrario. La poesia è un modo per riuscire a coinvolgere se stesso e il lettore con le immagini metaforiche, nel tentativo di salvare le illusioni. Nel Pensiero 259 l'entusiasmo e l'immaginazione, create dalle arti e dalla poesia, sono definite "opere di genio", essendo "la consolazione" dell'uomo a cui si contrappone a "l'infelicità della vita" (LEOPARDI, 1991, p. 227-228). È il tema centrale della poetica filosofica leopardiana, la poesia possiede l'energia e la forza per riuscire ad affrontare il dolore esistenziale, e anche se è un inganno impedisce che la verità provochi la fine alle illusioni. Ne La ginestra l'opera del genio è quella dei poeti, riesce a dare forza alla vita contro la visione della morte provocata dalla potenza distruttrice del vulcano. Il fiore emana un profumo che consola di fronte al deserto causato dall'eruzione vesuviana, espresso nei versi 32-37

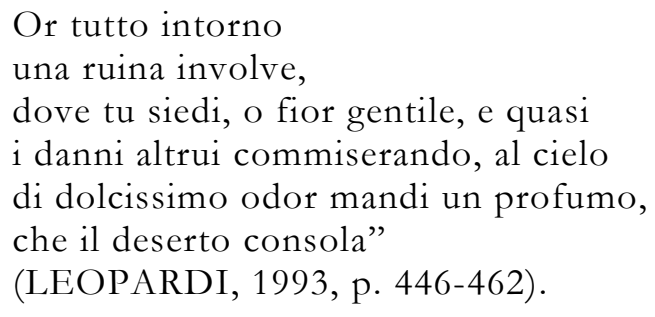

La bellissima illusione poetica è una metafora creata per suscitare sentimenti, immagini, emozioni, attraverso le quali il poeta desidera salvare se stesso e gli altri. In fondo è noto che la poesia, l'arte pittorica e la musica hanno un potere terapeutico che, in questo caso, potremmo attribuire alla poesia leopardiana; una funzione taumaturgica per guarire l'angoscia esistenziale dell'uomo moderno. Il potere salvifico delle illusioni è una bella invenzione per consolarsi e consolare. Per Leopardi "le opere di genio" sono la poesia e la filosofia e nel Canto della Ginestra 
illuminano la scena della nullità delle cose e l'infelicità della vita". Il "genio del deserto" sa e vede lo spettacolo del nulla e scopre la verità, ma sa imprimere energia e potenza al Canto con il dolce inganno dei versi.

Tuttavia se la natura è la nostra madre generatrice quando nasciamo, è anche crudele nel governarci "madre è di parto e di voler matrigna" (verso 125); a lei si contrappone l'alleanza degli uomini tra loro "confederati" che rappresentano l'umanità intera, schierata contro la natura crudele. È "l'umana compagnia" fin dall'inizi del mondo "in pria". L'immenso dolore universale viene addolcito dagli splendidi versi consolatori (vv.158-166)

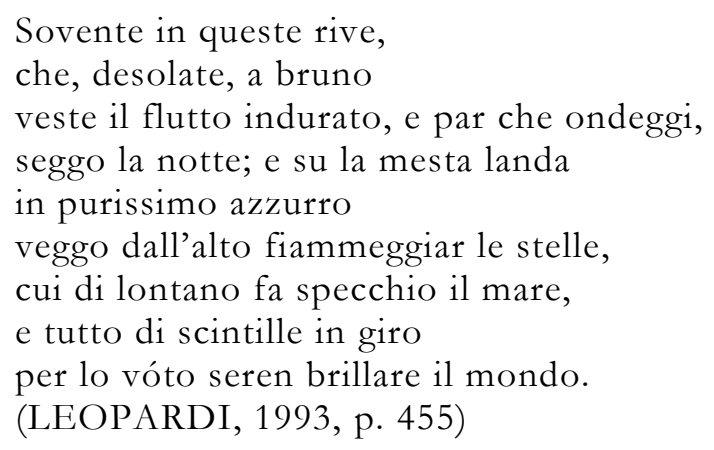

Si prosegue fino al verso 200 con un'immagine che si estende in uno spazio infinito tra il cielo, il mare, il mondo, le stelle e l'infinito spazio dell'universo, quasi impossibile alla comprensione dell'intelletto umano. Quando il dolore causato dalla natura nemica opprime suscitando terribili disperazioni nasce immediata la consolazione poetica che illumina i sentimenti e ravviva il cuore. Non ho dubbi nell'affermare che il potere taumaturgico dei versi ha una funzione terapeutica, perché creato dalle opere del genio che Leopardi descrive semplicemente come una strategia sperimentata nei confronti di se stesso e del lettore. La visione delle nullità genera stupore misto al 
senso di sgomento, eppure questa terribile immagine veritiera genera la vitalità dell'animo umano. Inoltre la forza suscitata rende il lettore soddisfatto di questa visione, seppur orrenda. Leopardi scrive nel Pensiero 260 e nel 261:

E lo stesso spettacolo della nullità, è una cosa in queste opere, che par che ingrandisca l'anima del lettore, la innalzi, e la soddisfaccia di se stessa e della propria disperazione. Gran cosa, è certa madre di piacere e di entusiasmo, e magistrale effetto della poesia, quando giunge a fare che il lettore acquisti maggior concetto di se, e delle sue disgrazie, e del suo stesso abbattimento e annichilamento di spirito. [...] Ma se questo sentimento è vivo, come nel caso ch'io dico, la sua vivacità prevale nell'animo del lettore alla nullità della cosa che fa sentire, e l'anima riceve vita [...] dalla stessa forza con cui sente la morte perpetua delle cose, e sua propria. (LEOPARDI, 1991, p. 228229).

Eppure da quanto si deduce dalla lettura del Pensiero 261 è un'invenzione poetica che apre altri orizzonti al nostro studio:

\begin{abstract}
Osserverò che il detto fenomeno occorre molto più difficilmente nelle poesie tetre e nere del Settentrione, massimamente moderne, come in quelle di Lord Byron, che nelle meridionali, le quali conservano una certa luce negli argomenti più bui, dolorosi e disperanti; e la lettura del Petrarca, per esempio de' Trionfi [...], dirò ancora di Verter, produce questo effetto molto più che il Giaurro, o il Corsaro ec. non ostante che trattino e dimostrino la stessa infelicità degli uomini, e vanità delle cose. [...] Io so che letto Verter $\mathrm{mi}$ sono trovato caldissimo nella mia disperazione letto Lord Byron, freddissimo, e senza entusiasmo nessuno; molto meno consolazione. (LEOPARDI, 1991, p. 229).
\end{abstract}

E' evidente che Leopardi sta parlando di un fenomeno letterario e narrativo, mediante il quale lo scrittore riesce a creare nel lettore immagini spaventose che suscitano stupore, o visioni sublimi che riescano ad appagare l'anima; in un certo senso l'autore racconta in prosa e in versi quello che il lettore vuole sentirsi narrare; Leopardi infatti pensa che solo pochi possiedano questa capacità poetico- 
narrativa, che per lui rappresenta uno strumento di riflessione filosofica.

\section{Poesia e filosofia}

La mia lettura nasce dall'analisi di Severino approfondita ne Il nulla e la poesia. Proporre un'esegesi in cui il pensiero filosofico sopravvive alla poesia è alla base dell'interpretazione. Il filosofo italiano ritiene che va considerato un altro aspetto importante: la poesia è forma e contenuto della filosofia. E' evidente che il pensiero filosofico espresso dal linguaggio poetico spiega il nesso imprescindibile tra filosofia e poesia, raggiungendo nei Canti i vertici più alti della sua liricità. La poesia, come contenuto e forma della filosofia, è un mezzo innocente, che crea illusioni salvifiche, ma svela freddamente la verità, quando l'uomo osa illuminare la realtà del nulla.

\footnotetext{
In lui, filosofia e poesia sono unite, non solo perché la poesia è il contenuto della filosofia, ma anche perché ne è la forma; non solo perché il filosofo nega l'illusione e il poetico della natura solo in quanto li conosce per propria esperienza, ma anche perché la poesia è la stessa grandezza e potenza del linguaggio - ossia dell'“opera" - che esprimendo la verità mantiene al di sopra del nulla. [...] la poesia non è solo il contenuto della filosofia, ma è il linguaggio che esprime con potente grandezza l'impotenza della nullità dell'uomo e di tutte le cose - il linguaggio "ardentissimo" del "freddissimo ragionatore. (SEVERINO, 2005, p. 312).
}

L'uomo riflette secondo ragione è un freddo calcolatore e distrugge le illusioni di cui invece ha bisogno. In questo caso la ragione è separata dalla poesia tanto che si può ipotizzare una dialettica di tipo hegeliana in cui l'approdo al nulla è il punto di arrivo del movimento dialettico. Il ragionamento esatto e freddo della "perfezione matematica" allontana "l'esistente", cioè l'uomo, dall'illusione e si rivela 
essere un metodo di analisi imperfetto, dunque la ragione matematica stessa è imperfezione (SEVERINO, 2005, p. 297).

L’ultrafilosofia è l'opera del genio? Il genio della poesia riesce a scoprire anche la possibilità di innalzare i popoli al di sopra dell'abisso del nulla grazie alle illusioni della poesia e sa unire la poesia alla filosofia: è ultrafilosofia. (SEVERINO, 2005, p. 329).

\section{La sorte della tecnica}

La ragione è la capacità di pensare attraverso cui l'uomo, può discernere e stabilire i rapporti logici tra le riflessioni e infine formulare i giudizi.

Nel Pensiero 115 Leopardi si chiede se il secolo in cui sta vivendo possa condurre al progresso e alla luce della verità profetizzata e speranza per il futuro. La ragione illuministica si rivela distruttrice perché conduce alla all'infelicità. La filosofia basata sull'uso della ragione distrugge le illusioni e $\mathrm{i}$ dolci inganni dei ricordi e della speranza. Nella poetica leopardiana poesia e filosofia hanno un rapporto logico inscindibile, essendo l'alternativa, espressa con "l'opera del genio" oggetto di riflessione nei Pensieri. La poesia possiede un'energia positiva che consente di uscire dal nulla nichilistico, cioè dal vuoto esistenziale. La ragione conduce l'uomo nell'abisso del dolore e del nulla, annientando la vitalità salvifica delle illusioni; inoltre l'uso della ragione illuministica è distruttiva per l'umanità perché annulla ogni speranza. Il genere umano raggiunge l'apice del movimento parabolico di discesa verso l'abisso nichilistico, fin tanto che nell'uomo resta viva la volontà di potenza. Quando infine essa viene negata non esiste più alcun segno vitale e subentra la noia leopardiana e il rischio di precipitare nell'abisso delle nullità. Di conseguenza Leopardi critica il 
movimento filosofico e culturale dell'illuminismo (l'età dei lumi), in quanto rappresenta per lui la fase distruttiva dell'umanità.

La civiltà della tecnica conduce l'uomo nel nulla nichilistico e la ragione è il metodo illuministico che distrugge, ingannando l'uomo con la falsa promessa di un finto progresso e benessere (Palinodia a Gino Capponi e La Ginestra) ${ }^{7}$. La prospettiva paradisiaca della scienza e della tecnica, basata sull'uso della ragione conduce l'uomo all'infelicità. Il pensiero umano fondato sull'analisi matematica e materialistica della ragione porta alla nullità delle cose, pertanto per Leopardi il pensiero illuminista è uno stato di calcolismo estremo (matematicità delle cose) che vanifica la stessa volontà di vita; un paradiso ingannatore attraverso il quale l'uomo si autodistrugge. L'analisi razionale di carattere matematico misura la realtà in modo esatto, mostrando la realtà, svelando la verità dell'essere: il nulla. (LEOPARDI, 1991, p. 15571558).

Cosa è l'uomo materialista e razionale senza sentimento poetico, senza l'immaginazione dei versi, senza le illusioni create dal dolce inganno della poesia? Per Leopardi è un eterno infelice che non vedrà mai la verità promessa dall'illuminismo. Nel Pensiero 2239 datato 11 luglio 1823, a proposito dei dogmi della Genesi e dell'albero della scienza e della Favola di Psiche, evidenzia la corruzione e la decadenza del genere umano per la presunzione di scoprire la verità, raggiungendo l'infelicità. Ma non sarà certo l'uso della ragione che permetterà di svelare il mistero dell'esistenza, perché il materialismo non garantisce di diventare sapienti, per quanto possa sembrare paradossale e contraddittorio, Leopardi ritiene che i veri sapienti di ogni epoca sono $i$ poeti, anche se mentono molto (Pensiero 2941). 
I primi sapienti furono i poeti, o vogliamo dire i primi sapienti si servirono della poesia, e le prime verità furono annunziate in versi, non, cred'io, con espressa intenzione di velarle e farle poco intelligibili, ma perché esse si presentavano alla mente stessa dei saggi in un abito lavorato dall'immaginazione, e in gran parte erano trovate da questa anzi che dalla ragione, anzi avevano eziando gran parte d'immaginario. (LEOPARDI, 1991, p. 1556).

Ebbene non solo l'immaginazione era il metodo dei poeti per svelare la verità, ma la poesia è sempre stata lo strumento migliore di riflessione dei saggi.

E inoltre per propria inclinazione e per secondare quella degli uditori, cioè de' popoli a cui parlavano, i saggi si servivano della poesia e della favola per annunziar la verità, benché niuna intenzione avessero di renderle méconnaissables. (LEOPARDI, 1991, p. 1557)

Leggendo i Pensieri si ha la certezza che Leopardi scriveva senza voler essere nichilista; infatti credo che si tratti di un intellettuale versatile, talvolta poeta, prosatore satirico, sociologo, e soprattutto un filosofo aperto a tutte le sollecitazioni culturali del tempo e perfino alle mode. La motivazione delle riflessioni sui temi esistenziali fa pensare che fosse ispirato da un'indole sensibile, capace di renderlo anticipatore inconsapevole del nichilismo. Eppure è senza dubbio il primo filosofo poetante anticipatore del nichilismo, scopritore del movimento parabolico che nasce dal nulla per poi ritornare nel movimento vitale infinito dell'universo. Quale meraviglioso e migliore inganno esiste nella nostra natura umana, se non la poesia e la filosofia? Scrive nel Pensiero 3283 che "il vero poeta è sommamente disposto ad esser gran filosofo, e il vero filosofo ad esser gran poeta" se usano l'immaginazione (LEOPARDI, 1991, p. 1770-1771). E quindi “La poesia e la filosofia sono entrambe del pari, quasi la sommità 
dell'umano spirito, le più nobili e le più difficili facoltà a cui possa applicarsi l'ingegno umano". (LEOPARDI, 1991, p. 1771).

\section{Riferimenti bibliografici}

BINNI, Walter. Nuova poetica leopardiana. Firenze: Sansoni, 1984.

LEOPARDI, Giacomo. Canti, DOTTI, Ugo (Org.), Milano: Feltrinelli, 1993.

- Zibaldone di Pensieri, PACELLA, Giuseppe

(Org.), 3 volumi. Milano: Garzanti, 1991.

Feltrinelli, 1993. . Operette morali, PRETE, Antonio (Org.). Milano: Lettere. Milano: Mondadori, 1993.

LUPORINI, Cesare. Leopardi progressivo. Roma: Editori Riuniti, 2018.

NEGRI, Antimo. Interminati spari ed eterno ritorno. Nietssche e Leopardi. Firenze: Le Lettere, 1994

PRETE, Antonio. Il pensiero poetante. Saggio su Leopardi. Milano: Feltrinelli, 1997. Finitudine e infinito. Su Leopardi. Milano: Feltrinelli, 1998.

SEVERINO, Emanuele, L'essenza del nichilismo. Milano: Adelphi Edizioni, 1995. . Il nulla e la poesia. Milano: Rizzoli BUR, 2005.

Rizzoli, 2006.

Cosa arcana e stupenda. L'Occidente e Leopardi. Milano: . In viaggio con Leopardi. La partita sul destino dell'uomo. Milano: Rizzoli, 2015. 
TIMPANARO, Sebastiano. Alcune osservazioni sul pensiero di Leopardi, in "Classicismo e illuminismo nell'Ottocento italiano". Pisa: Nistri-Lischi, 1984, pp. 133-182.

VERRI, Pietro. Discorso sullindole del piacere e del dolore. Londra: Traiettiana, 2010.

\begin{abstract}
Notas
${ }^{1}$ Emanuele Severino, recentemente scomparso dopo una lunga vita di studi è considerato uno dei filosofi italiani ed europei più importanti della nostra epoca. Di origine siciliana e bresciana, ha trascorso molti anni in studi intensi sulla filosofia Occidentale da lui ritenuta impregnata profondamente dal Nichilismo. La sua bibliografia è immensa spazia dagli studi filosofici su Johann Gottlieb Fichte a un salvifico ritorno alla filosofia di Parmenide. Ha dedicato a Leopardi tre saggi: Il nulla e la poesia, Cosa arcana e stupenda e In viaggio con Leopardi.

${ }^{2}$ Con il termine "Essere" si vuole indicare il concetto di Parmenide espresso ne Sulla natura.

${ }^{3}$ La fonte delle citazioni dei Pensieri dello Zibaldone è l'edizione di Giuseppe Pacella che riunisce interamente i Pensieri in tre volumi pubblicati nel 1991 (LEOPARDI, 1991).

${ }^{4} \mathrm{Nel}$ pensiero di Eraclito il logos è la legge universale secondo la quale tutte le cose accadono, determinando l'armonia degli opposti. L'ordine è visto come un fuoco eterno vivente che si ravviva o si spegne secondo le trasformazioni dei vari stati della natura. Si pensi all'espressione del "panta rei": tutto scorre, "non potrai bagnarti due volte nelle acque dello stesso fiume"; ciò significa che ogni elemento degli opposti si trasforma nell'altro (dottrina del fuoco). I contrari si scambiano l'uno con l'altro secondo giustizia. La giustizia degli eventi avviene per contrasto e necessità, ma anche per il conflitto universale (il polemos). Quindi Eraclito, filosofo del divenire, si contrappone a Parmenide, filosofo dell'essere immutabile. Si ricordi soprattutto che i greci spiegano tutto con il divenire, perché cercano una fonte cosmica da cui tutte le cose sono state create.

${ }^{5}$ Sono i versi 17-20 di La ginestra o il fiore del deserto, scritta nel 1836; durante il periodo trascorso da Leopardi a Napoli, nella Villa Ferrigni, proprio nei pressi del Vulcano Vesuvio, su indicazione del critico letterario Ugo Dotti. (DOTTI, 1993).

${ }^{6}$ Leopardi indicava con l'espressione Idee l'Iperuranio di Platone.

${ }^{7}$ Severino conduce un'analisi interessante dei versi dei canti La palinodia e La ginestra, i due canti contengono lo sviluppo in versi di un aspetto importante del pensiero filosofico leopardiano: il paradiso della tecnica, promesso dall'era dei lumi con "le magnifiche sorti progressive". Cfr. G. Leopardi, La ginestra, in cit. Per un approfondimento di questa tematica rinvio ai capitoli del saggio di Severino Il nulla e la poesia, dedicati alla "degradazione del secolo XIX"; cfr. E. Severino, cit., pp. 175-231, pp. 272-277 e infine pp. 323-346. Si tratta di pagine efficaci dal punto di vista filosofico che meritano ulteriore approfondimento e dovrebbero essere oggetto di maggiore riflessione e di dibattito per i filosofi contemporanei del nostro tempo, per discutere su dove sta andando il finto progresso di oggi, e per comprendere se siamo di fronte alla degradazione del secolo XXI.

${ }^{8}$ Irriconoscibile, incommensurabile, irreconhecível.
\end{abstract}

